\title{
Factors associated with birth weight inequalities in Jordan
}

\begin{abstract}
Background: Birth weight (BW) is a good indicator of mothers and neonates nutritional status, and it contributes to the newborn baby's survival, health, growth, and development. Aim: To identify social factors associated with differences in mean birth weight of newborn babies in Jordan. Methods: This retrospective study analyzed medical records to determine possible risk factors associated with differences in newborn birth weight in the Irbid governorate of Jordan. All full-term singleton births during the year 2010 were reviewed. Abstracted data included mother's age and educational level, and monthly family income. Newborn information included birth weight, gender and birth order. Results: A total of 5414 full term singleton births were included. Of these 818 (15.1\%) were low birth weight, 3984 (73.6\%) were normal birth weight, and $612(11.3 \%)$ were high birth weight. Bivariate analysis of variance revealed that low mean BW was associated with female gender, first birth babies, higher maternal age ( $>35$ years), lower educational level, and lower income $(<500 \mathrm{JD})$. Multivariate analysis of variance revealed that mean BW was lower in female infants, first born infants, infants of less educated mothers, higher age, and low monthly income. Limitations: The findings can be generalised to fullterm singleton pregnancies in countries who share similar cultural and traditional values. Conclusion: Education of mothers is a modifiable variable that can positively influence BW, particularly in the case of female and first born infants.

Implication for Nursing and Health Policy: The findings inform our understanding of some social factors affecting $\mathrm{BW}$ of neonates in Jordan and development of effective public health interventions that could reduce the adverse effects of such factors on newborn birth weight. Preconception and antenatal care is also important
\end{abstract}


for early detection of such possible risk and targeting mothers who require early interventions and support.

Key words: birth inequality, low birth weight, singleton births, maternal age, gender, education, socioeconomic status, Middle East 


\section{Introduction}

Birth weight $(\mathrm{BW})$ is a good indicator of mothers and neonates nutritional status, and it contributes to the newborn baby's survival, health, growth, and development (Gage et al. 2013; Risnes et al. 2011;Roberts et al. 2006). Birth weight is also recognized as an important measure of mortality risk during the infancy period (Gage et al. 2013; Risnes et al. 2011;United Nations Childrens Fund and World Health Organization 2004).

Infant birth weight is dependent on gestation and rate of fetal growth (Emamghorashi $\&$ Heydari 2008). Both low birth weight $(\mathrm{BW}<2500 \mathrm{~g})$ and high birth weight $(\mathrm{BW}>$ $4000 \mathrm{~g})$ are associated with different short and/or long-term health problems. These include, but are not limited to: delays in childhood motor, cognitive and social development, birth injuries, obesity and chronic diseases later in the child's life (Halileh et al. 2008; Lawn et al. 2010). It is, therefore, important to identify factors that could affect birth weight.

Factors associated with low birth weight include: preterm birth (Abdul-Kader 2010; Carlo et al. 2011), being a girl or first child (Al-Akour 2008; Bisai et al. 2006; Dubois \& Girard 2006; Halileh et al. 2008), low maternal weight gain during pregnancy (Bisai et al. 2006), poor maternal nutritional status (Al-Akour 2008; Brettell et al. 2008; Das \& Paul 2008), maternal age $(<20$ years) (Bisai et al. 2006), low body mass index (BMI) before pregnancy (Upadhyay et al. 2011; Ye et al. 2010), low socioeconomic status (Brown et al. 2002; Das \& Paul 2008; Mohammadzadeh et al. 2010), and maternal smoking (Langley et al. 2007; Ward et al. 2007). Factors particularly associated with high birth weight include: higher maternal BMI before 
pregnancy (Upadhyay et al. 2011; Ye et al. 2010), high weight gain during pregnancy (Monte et al. 2011), and gestational diabetes (James 2001).

Both low and high birth weight may be related to social factors such as: poor access to maternity care before and during pregnancy, poor maternal health knowledge, environmental factors such as poverty and overcrowding, as well as poor health education and advice (Khalaf et al. 2007). As such, birth weight inequality is an important area of public health research. Examining the spectrum of birth weight (low, normal and high) is important to determine factors effecting newborn birth weight (Dubois \& Girard 2006). Furthermore, identifying important social factors influencing birth weight will assist in the development of public health interventions to promote a good start to life. There is, however, a dearth of research on birth weight inequalities from Middle Eastern countries. To our knowledge, this study is the first to explore social factors associated with differences in newborn birth weight in Jordan.

\section{Routine antenatal care in Jordan}

Infant mortality rate in Jordan is 23 per 1000 live births (Department of Statistics and Macro International Inc. 2010). Recent data indicates that $99 \%$ of Jordanian women receive antenatal care from qualified staff with the majority of them (93\%) receiving the care from a doctor and others receiving care from a midwife or a nurse (Al-Akour 2008).

Around $81 \%$ of Jordanian women have more than five antenatal care (ANC) visits but the percentage of visits is lower in rural areas than urban areas (Al-Akour 2008). Eighty-five percent of women have their first ANC visit before the fourth month of pregnancy which means that most of Jordanian women start ANC at a relatively early 
stage of their pregnancy (Department of Statistics and Macro International Inc. (DSMII) 2010).

There are ultrasound facilities in most clinics which doctors use at every visit to monitor fetal growth, confirm the location of the placenta, detect number of fetuses, and birth abnormalities. Furthermore, ultrasound is sometimes used for the only purpose of fetal sex detection. Doctors also request blood and urine samples if required. Nurses usually measure a woman's blood pressure and weight at each visit. Electronic fetal monitoring is performed using a cardiotocograph (CTG). Midwives perform a CTG if requested by the doctor, however, decisions regarding the CTG results are made by the doctor.

In Jordan, there is a remarkable shortage in the provision of basic maternity care. A limited application of evidence-based practice, a lack of health education and counseling for women and families including information about healthy lifestyles have been noted (Abushaikha 2007; Khalf et al. 2007; Mohammad et al. 2011).

\section{Method}

A retrospective chart audit of all full-term singleton pregnancies at the Princess Badeah Teaching Hospital was conducted between 1 January and 31 December 2010. Princess Badeah Teaching Hospital is government funded and the largest maternity hospital in Irbid governorate of Jordan, with an annual birth rate of around 10,000 births.

It is routine practice to weigh infants at birth and document this. Abstracted data included demographic details such as maternal age, educational level, gravidity, and monthly income of the family. Newborn information includes gender and birth 
weight. Maternal health records with no values for newborn birth weight were disregarded. The study was approved by the Institutional Review Board at Jordan University of Science and Technology and the Ministry of Health in Jordan.

Data analysis was performed with SPSS version 17. Bivariate analysis was performed first to explore the associations between birth-weight (dependent variable) and the independent variables. Variables affecting birth-weight on the bivariate analysis of variance were subsequently evaluated by multivariate analysis of variance. A multivariate with the stepwise procedure was then used to identify the best independent variables affecting birth-weight. The relative contribution of each factor to the birth weight was described using the coefficient of determination $\left(\mathrm{R}^{2}\right)$ from the multivariate model. The independent variables were newborn gender and birth order, mothers' age and educational level, and monthly income of the family $(<500 \mathrm{JD}$ (low), $500-1000 J D$ (Medium), or $>1000 J D$ (high)). Significance was a $p$-value less than 0.05 .

\section{Results}

Of the 6072 singleton births during the study period, 5414 (89.2\%) full term births were eligible for the study. Six hundred fifty-eight (10.8\%) were excluded because they were preterm babies and/or multiple pregnancies. The mean birth weight for newborns was 3241 grams. Table 1 presents the distribution of newborn birth weights. A total of $15.1 \%$ of newborns had low birth weights $(<2500 \mathrm{gm}), 73.6 \%$ had normal birth weights $(2500-4000 \mathrm{gm})$, and $11.3 \%$ had high birth weights $(>4000 \mathrm{gm})$.

More than half of the mothers (56.4\%) were 25 - 35 years old and $56 \%$ had completed higher education compared to $9.3 \%$ who had low education (9 years compulsory school). Less than a third (30.2\%) of mothers reported high monthly 
family incomes (> 1000 Jordanian Dinar (JD)), 56.4\% reported medium monthly income (500-1000JD), and the rest (13.4\%) reported low monthly incomes $(<500 \mathrm{JD})$. The sample had slightly less female infants than males $(48.7 \%$ vs. $51.3 \%)$, with $57.5 \%$ of newborns being first born.

Table 2 shows the bivariate association between infants' mean birth weight (grams) (dependent variable) and independent variables. Mean birth weight was lower in female babies $(\mathrm{F}=85.88, \mathrm{P}<0.001)$, first born babies $(\mathrm{F}=5.89, \mathrm{P}=0.015)$, higher maternal age (more than 35 years) $(\mathrm{F}=13.74, \mathrm{P}<0.001)$, lower educational level $(\mathrm{F}$ $=51.86, \mathrm{P}<0.001)$, and lower monthly income $(\mathrm{F}=21.79, \mathrm{p}<0.001)$.

The best multivariate model that associated with low mean birth weight included female infants $(\mathrm{F}=94.67, \mathrm{P}<0.001)$, first born infants $(\mathrm{F}=16.19, \mathrm{P}<0.001)$, infants of less educated mothers $(\mathrm{F}=37.63, \mathrm{P}<0.001)$, infants of higher age mothers $(\mathrm{F}=$ $7.28, \mathrm{P}<0.001)$, and infants of mothers with low monthly income $(\mathrm{F}=21.27, \mathrm{P}<$ 0.001). This multivariate model predicted $13 \%$ of the variance. The largest association with low mean birth weight was infants of mothers with less education $\left(\mathrm{R}^{2}=0.023\right)$, followed by female infants $\left(\mathrm{R}^{2}=0.017\right)$, infants of mothers with low monthly income $\left(\mathrm{R}^{2}=0.007\right)$, and first born infants $\left(\mathrm{R}^{2}=0.001\right)$ (Table 3).

\section{Discussion}

This is the first study to investigate factors associated with birth weight inequalities in Jordan. The major strength of the study is collection of the required data through birth records (Halileh et al. 2008; O'Sullivan et al. 2000). The results contribute to a better understanding of perinatal outcomes in Jordan. 
The gender differences in mean birth weight are slightly higher than previously reported in a study in Kuwait (Alshimiri et al. 2003) but lower than those reported in a Palestinian study (Halileh et al.2008). In the present study, mean birth weight was lower in female infants and this is similar to results of previous studies conducted in the Middle East, India, and China (Alshimiri et al. 2003; Halileh et al. 2008; Al-Qutob et al. 2004; Shah 2010). Al-Qutob et al.(2004) conducted a study with 1195 Jordanian women to investigate the effect of prenatal knowledge about foetal gender on infant mean birth weight. They found that women carrying male infants had higher mean birth weights compared with women carrying female infants. Another Jordanian study conducted by Al-Akour (2008) found that when the gender of foetus was known, the mean number of antenatal care visits was significantly higher for women pregnant with male foetuses than that of women pregnant with female foetuses.

Socio-cultural factors influence gender preference and can have potentially detrimental outcomes in Jordanian society. In Arab culture, gender differences in the perceived value of children exist and daughters are less valued than sons (Mohammad et al. 2011). This view is linked to the potentially lower economic productivity of girls compared to boys. Remittances to parents are lower from daughters than sons, males sustain the family line, and provide for parents in their old age (Al-Qudsi1998; Mohammad et al. 2011).Because sons are perceived to be of more value than daughters in this culture, women, their partners and extended families tend to give more attention to the woman's health and well-being when pregnant with a male baby. This additional care can include better food and nutrition and can result in higher birth weight. In the present study, first born babies tended to have a low mean birth weight. This may due to a mother's first experience of pregnancy and poor health knowledge on care of herself and her unborn baby (Lin et al. 2011). Results of 
the current study are consistent with other studies which reported that mean birth weight of first born babies is lower than mean birth weight of infants born to multiparous women (Lin et al. 2011). Another study conducted in India showed that regardless of maternal age, the mean birth weight was much lower in first born babies than later birth orders (Maitra et al. 1995).

The finding that the mean birth weight of newborns increased as maternal education increased was also supported by previous studies (Halileh et al. 2008, Muula et al. 2011; Siza 2008). Previous results indicated that mean birth weight was lower in infants of mothers with no formal education than infants of mothers who attained higher education (Muula et al. 2011;Siza 2008). Well-educated mothers tend to have more knowledge about appropriate pregnancy care and are more aware of the importance of antenatal care to them and their unborn child which may positively affect pregnancy outcomes (Siza 2008). It also could be that by increasing mothers' education the likelihood of low birth weight is reduced as mothers are more aware about pregnancy risks (Cage et al. 2013).

Low mean birth weight was also associated with advanced maternal age ( $>35$ years). This may be related to the fact that this age ( $>35$ years) is measured as the threshold age for foetus and mother health risk (Astolfi \& Zonta 1999). Several studies support this finding by showing that low birth weights of newborns were associated with advanced maternal age (Das \& Paul 2008; Khoshnood et al. 2005; Newburn-Cook \& Onyskiw 2005).

In the current study, low mean birth weight was associated with low total monthly income. This is consistent with previous studies that showed an association between birth weight and total monthly income(Muthayya 2009). Low total monthly income 
has been found to directly affect maternal nutrition and attendance at antenatal care (Das \& Paul 2008;Muthayya 2009; Shah \& Ohlsson 2010) which will indirectly affect the birth weight of her child.

\section{Implication for Nursing and Health Policy}

The findings of current study help in understanding related social factors affecting birth weight of neonates in Jordan. These findings should be considered by policy makers and prompt the development of effective public health interventions that could reduce the adverse effects of such factors on newborn birth weight. Health care providers could play an important role in improving outcomes for Jordanian women and their families. Monitoring of individual-level factors such as maternal age, parity and infant gender are important to recognise and openly discuss with women as part of their ongoing health education that aim to increase newborn birth weight. Preconception and antenatal care is also important for early detection of risk and targeting mothers who require early interventions and support. For example, nurses and midwives could develop culturally sensitive educational materials, and prevention strategies about low weight gain and other topics in maternity health care settings. Appropriate interventions should be implemented for early detection and follow-up of high-risk mothers.

Further studies should be conducted to explore the content of maternity care programs in Jordan in order to identify which components are more effective to achieve good health outcomes and the best way to implement these programs (such as groups and individualized interventions, face-to-face education, and use of printed formats). 
Moreover, future studies must be conducted to evaluate the quality of care provided and its effects on the development of different pregnancy outcomes.

\section{Strengths and Limitations}

The current study was conducted with a large representative sample of full-term singleton pregnancies to assess social factors associated with differences in newborn birth weight in Jordan. The findings can be generalised to most full-term singleton pregnancies in countries who share similar cultural and traditional values. However, other variables influencing birth weight reported in the previous studies were not available for the current study, such as maternal weight, biological factors, complications during pregnancy, and negative maternal practices such as smoking.

\section{Conclusion}

The current study confirmed results of earlier research which reported an association between some maternal and fetal risk factors, and differences in newborn birth weight. The plausibility of some associations between some factors such as baby's gender, birth order, mother's age, mother's education, and mother's total monthly income were significant in infants' low mean birth weight. This is the first published study in Jordan to show such associations between risk factors and newborn birth weight. These study findings are important as they can lead to improvements in maternal and infant health. 


\section{References}

Abdul-Kader, S.(2010)Preterm delivery in association with maternal age, birth order and fetal gender.Unpublished PhD Thesis. Degree of Fellowship in Obstetrics and Gynaecology. Iraqi Board for Medical Specializations (Iraq).

Adams, M., Li, H., Ogburn, L. \& Danilenko-Dixon, R. (1998) Sequelae of unrecognisedgestational diabetes. American Journal of Obstetrics and Gynecology,178(1),1321-1332.

Ahmed, U., Das, M. \& Mostafa, G. (1994) Association of maternal biological factors with birth weight in Bangladesh. Journal of Preventive and Social Medicine, 13(2), $52-57$.

Al-Akour, N.(2008) Knowing the fetal gender and its relationship to seeking prenatal care: results from Jordan. Journal of Maternal and Child Health,12(2), 787-792.

Al-Qudsi, S.(1998)The demand for children in Arab countries: evidence from panel and count data models. Journal of Population Economics, 11(3), 435-452.

Al-Qutob, R., et al. (2004)The effects of prenatal knowledge of fetal sex on birth weight: a study from Jordan. Health Care for Women International, 25(3),281-291

Astolfi, P. \& Zonta, A.(1999) Risks of preterm delivery and association withmaternal age,birth order, and fetal gender. Human Reproduction, 14(11), 2891-2894.

Bisai, S., et al.(2006) The effect ofmaternal ageand parity on birth weight among Bengalees of Kolkata, India.Ecology, Culture, Nutrition, Health and Disease, 14(3), 139-143.

Brettell, R., Yeh, S. \& Impey, W.(2008) Examination of the association betweenMalegender and preterm delivery. European Journal of Obstetrics and Gynecology Reproductive Biology, 141(3), 123-126.

Brown, E., Murtaugh, A., Jacobs, R. \& Margellos, C.(2002) Variation in newbornsizeaccording to pregnancy weight change by trimester. Amirican Journal of Clinical Nutrition, 76, 205-209.

Carlo, A., McDonald, A. \& Fanaroff, A.(2011) Association of antenatalcorticosteroidswith mortality and neurodevelopmental outcomes among 
infants bornat 22 to 25 weeksgestation. Journal of American Medical Association, 306(21), 2348-2358.

Das, J. \& Paul, N. (2008) Association of maternal socio-biological factors with birth weight of newborn babies. Journal of Chittagong Medical College Teachers'Association, 19(1), 37-42.

Department of Statistics [Jordan]. (2010) Jordan Population and Family Health Survey 2009. Maryland, USA: Department of Statistics and ICF Macro.

Department of Statistics [Jordan]. (2003) Jordan Population and Family Health Survey, 2002. Maryland, USA: Department of Statistics and ICF Macro.

Dubois, L. \& Girard, M. (2006) Determinants of birth weight inequalities:populationbasedstudy. Pediatrics International, 48(2), 470-478.

Dunger, D., Petry, C. \& Ong, K. (2007)Genetics of size at birth.Diabetes Care,30(2),S150-S155.

Emamghorashi, F. \& Heydari, S. (2008) Neonatal birth weight and related factors inSouthof Iran, Johrom. Journal of Family and Reproductive Health, 2(4), 191193.Gage, T., Fang, F., O'Neill, E. \& DiRienzo, G. (2013) Maternal education, birth weight, and infant mortality in the United States. Demographic, 50(2), 615-635.

Halileh, S., et al. (2008)Determinants ofbirth weight:gender based analysis. Maternal and Child HealthJournal,12(1), 606-612.

James, H. (2001) Gestational Diabdetes, birth weight, sex ratio, and cesarian section.Diabetes Care,24(11), 2018-2019

Khoshnood, B., Wall, S. \& Lee, S. (2005) Risk of low birth weight associated withadvanced maternal age among four ethnic groups in the United States. Maternal and ChildHealthJournal,9(1), 3-9.

Langley, K., Holmans, P., Bree, M. \& Thapar, A. (2007) Effects of low birth weight,maternal smoking in pregnancy and social class on the phenotypic manifestation ofAttentionDeficit Hyperactivity Disorder and associated antisocial behaviour:investigation in a clinicalsample. BMC Psychiatry, 7(3), 26-32.

Lawn, E., et al. (2010)Kangaroomother care to prevent neonatal deaths due to preterm birth complications.InternationalJournal of Epidemiology, 39(1), 144-154. 
Mohammad, K., Gamble, J.\& Creedy, D K.(2011) Prevelance and factors associated with the development of antenatal and postnatal depression among Jordanian women. Midwifery,27(6), 238- 245.

Mohammadzadeh, A., Farhat, A., Amiri, R. \& Esmaeeli, H. (2010) Effect of birth weightand socioeconomic status on children's growth in Mashhad, Iran.International Journal ofPediatrics.Article ID 705382, 5 pages. Available at: http://dx.doi.org/10.1155/2010/705382

Monte, S., Valenti, O., Giorgio, E. \& Renda, E. (2011) Maternal weight gain duringpregnancy and neonatal birth weight: a review of the literature. Journal of Prenatal Medicine, 5(2),27-30.

Muthayya, S. (2009) Maternal nutrition \& low birth weight - what is reallyimportant?Indian Journal of Medical Research,130(1),600-608.

Muula, S., Siziya, S. \& Rudatsikira, E. (2011) Parity and maternal education are associatedwith low birth weight in Malawi. African Health Science, 11(1),65-71.

Newburn-Cook, C. \& Onyskiw, J. (2005) Is older maternal age a risk factor forpreterm birth and fetal growth restriction? Health Care for WomenInternational, 26(8), 852-875.

Risnes, K., et al. (2011) Birth weight and mortality in adulthood: a systematic review and meta-analysis. International Journal of Epidemiology, 40, 617-661.

Roberts, G., Bellinge, RD. \& McCormick, C. (2006) A cumulative risk factor model for early identification of academic difficulties in premature and low birth weight infants. Journal of Maternal and Child Health,11(2), 161-172.

Shah, P. \& Ohlsson, A. (2010) Paternal factors and low birth weight, preterm, andsmall forgestational age births: a systematic review. American Journal of Obstetrics and Gynecology, 202(2),103-123.

Siza, E.(2008) Risk factors associated with low birth weight of neonates amongPregnantwomen attending a referral hospital in northern Tanzania. Tanzania Journal of HealthResearch, 10(1), 1-8.

UNICEF. (2013) Jordan - Statistics. Retrieved 10/05/2014, from http:// www.unicef.org/inforbycountry/jordan_statistics.html 
United Nations Childrens Fund and World Health Organization. (2004) Low birth weight:Country, regional and global estimates. Geneva: WHO.

Upadhyay, S., et al. (2011) Association between maternal body mass index and the birth weight of neonates. Journal of Nepal Medical College, 13(1),42-45.

Ward, C., Lewis, S. \& Coleman, T. (2007) Prevalence of maternal smoking and environmental tobacco smoke exposure during pregnancy and impact on birth weight: retrospective study using Millennium Cohort. BMC Public Health, 7, 81-87.

Yang, S., Lynch, J. \&Ezra, S. (2008)Birthweight and cognitive ability in childhood among siblings and nonsiblings. Pediatrics, 122(8), 350-361.

Ye, R., et al. (2010) Birth weight, maternal body mass index, and early childhood growth: aprospective birth cohort study in China. Journal of Epidemiology,20(6), 421-428. 
Table1: Infants' birth weights compared to national data

\begin{tabular}{lcc}
\hline Birth weight & No (\%) & $\begin{array}{c}\text { Comparison with Jordanian } \\
\text { birthing population }\end{array}$ \\
& & No (\%) \\
\hline Low birth weight $(<2500 \mathrm{gm})$ & $818(15.1)$ & $(31515) 16.5$ \\
Normal birth weight $(2500 \mathrm{gm}-4000 \mathrm{gm})$ & $3984(73.6)$ & $(151081) 79.1$ \\
High birth weight $(>4000 \mathrm{gm})$ & $612(11.3)$ & $(8404) 4.4$ \\
Total & $5414(100)$ & $191000(100)$ \\
\hline
\end{tabular}

Table2: Bivariate relationship between infants' mean birth weight (grams) and categorical variables

\begin{tabular}{lccccc}
\hline Factor & No (\%) & Mean(SD) & df & P & F \\
\hline Baby's gender & & & 1 & & 85.88 \\
Male & $2775(51.3)$ & $3304.86(476.70)$ & & & \\
Female & $2639(48.7)$ & $3173.54(515.99)$ & & $<0.001^{*}$ & \\
Birth order & $3112(57.5)$ & $3222.42(516.26)$ & & $0.015^{*}$ & \\
First & $2302(42.5)$ & $3265.77(477.39)$ & & & \\
Second or more & $1559(28.8)$ & $3228.67(438.61)$ & & & \\
Mother's age & $3055(56.4)$ & $3270.77(479.13)$ & & & \\
Less than 25 years & $800(14.8)$ & $3150.35(657.78)$ & & $<0.001^{*}$ & \\
Between 25 and 35 years & & & 4 & & 51.86 \\
More than 35 years & $505(9.3)$ & $2944.85(752.13)$ & & $<0.001 *$ & \\
Mother's educational level & $1879(34.7)$ & $3240.94(439.49)$ & & & \\
Low (less than 9 yrs compulsory schooling) & $1453(26.8)$ & $3273.37(429.30)$ & & & \\
High school & $1076(19.9)$ & $3280.86(477.54)$ & & & \\
Diploma & $501(9.3)$ & $3358.68(531.03)$ & & \\
Bachelor & & & & \\
Above bachelor level & $726(13.4)$ & $3180.03(464.94)$ & & $<0.001 *$ & \\
Total monthly income & $3051(56.4)$ & $3223.51(532.45)$ & & & \\
Low (<500JD) & $1637(30.2)$ & $3300.15(445.91)$ & & & \\
Medium (500-1000JD) & & & & \\
High $(>1000 J D)$ & & & & & \\
Error = 5403 & &
\end{tabular}


Table3: Multivariate linear model (stepwise stepping procedure) for differences in mean birth weight of newborn babies

\begin{tabular}{lccc}
\hline Factors & B coefficients & P value & Partial $\mathbf{R}^{\mathbf{2}}$ \\
\hline Infants of mothers with less education & 0.148 & $<0.001$ & 0.023 \\
Female infants & 0.130 & $<0.001$ & 0.017 \\
Infants of mothers with low monthly income & 0.083 & $<0.001$ & 0.007 \\
First born infants & 0.036 & 0.007 & 0.001 \\
\hline
\end{tabular}




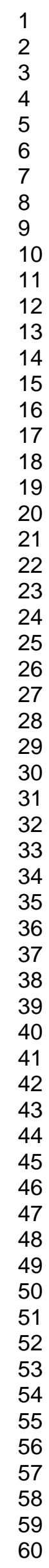

2

4

5

7

8

10

11

12

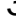

15

16

17

19

20

21

23

24

26

27

29

30

33

34

35

36

37

39

40

41

42

44

45

46

47

48

49

51

52

55

56

57

59

60 
Response to reviewers

Editor's comments

- In putting your manuscript through the software I have found there are several instances where there are long strings of words/phrases that require appropriate referencing, either using the direct quote method (author, year page number) or by rephrasing in your own words and giving the author and year in brackets.

Please carefully look at attached file, and the crossreferencing in color with the corresponding list of references at the back of the manuscript that has been - we have carefully looked at the attached file you sent it to us and also at the crossreferencing in color with the corresponding list of references, and we have rephrased the sentences in our own words and gave the author and year in brackets. generated through Cross-Check. 